LAEFF-94/15

\title{
BIG-BANG NUCLEOSYNTHESIS AS A PROBE OF THE GEOMETRY OF SUPERSPACE
}

\author{
A. Llorente and J. Pérez-Mercader \\ Laboratorio de Astrofísica Espacial y Física Fundamental \\ Apartado 50727 \\ 28080 Madrid (Spain)
}

\begin{abstract}
We study the effect from a general ultralocal supermetric on primordial nucleosynthesis for Friedmann-Robertson-Walker Cosmology. The parameter $\lambda$ of the supermetric changes the effective number of degrees of freedom, $g_{*}$, which modifies the Friedmann equation. This modification produces variations in the production of primordial ${ }^{4} \mathrm{He}$. The observations of the primordial abundances of light elements $\left({ }^{4} \mathrm{He}\right.$, $\mathrm{D},{ }^{3} \mathrm{He}$ and ${ }^{7} \mathrm{Li}$ ) allow to estimate bounds on the values of $\lambda$ consistent with these observations. We obtain $0.87 \leq \lambda \leq 1.04$. In addition we analyze the importance of $\lambda<1$ to explain possible incompatibilities in the standard Big-Bang nucleosynthesis.
\end{abstract}


In the first quantization of gravity 1$]$ one needs to introduce a "metric of metrics", the supermetric $G_{i j k l}$. The most general ultralocal supermetric depends on a free parameter $\lambda$, which is usually fixed, on arbitrary grounds, to $\lambda=1$. For second quantized gravity, the value of $\lambda$ appears, e.g., on the $\beta$-functions for Newton's Constant and the Cosmological Constant [2], the two dimensionful parameters in the four dimensional gravitational action.

In this paper, we study the effect of $\lambda$ on cosmological light element production and show how the observational values of the abundances of ${ }^{4} \mathrm{He}$, $D,{ }^{3} \mathrm{He}$ and ${ }^{7} \mathrm{Li}$ can be used to bound $\lambda$.

Let us consider General Relativity (GR) in its 3+1 ADM formulation [3]. The hamiltonian $H$ is

$$
H=\int d^{3} x\left(N H_{G}+N_{i} H^{i}\right)
$$

where the hamiltonian constraint is

$$
H_{G} \equiv 16 \pi G G_{i j k l} \pi^{i j} \pi^{k l}-\frac{\sqrt{h}^{3} R}{16 \pi G}
$$

with the supermetric given by

$$
G_{i j k l} \equiv \frac{1}{2 \sqrt{h}}\left(h_{i k} h_{j l}+h_{i l} h_{j k}-h_{i j} h_{k l}\right)
$$

and where $h_{i j}$ is the induced spatial metric, $N$ is the lapse function and $N^{i}$ the shift vector. $G$ is Newton's Constant. The $\pi^{i j}$ are the momenta conjugate to $h_{i j}$.

In the presence of matter the constraint equation, $H_{G}=0$, becomes

$$
16 \pi G G_{i j k l} \pi^{i j} \pi^{k l}-\frac{\sqrt{h}^{3} R}{16 \pi G}-T=0
$$

where $T$ is the $T^{0}{ }_{0}$ component of the stress-energy tensor of the matter fields.

For a Friedmann-Robertson-Walker metric

$$
d s^{2}=d t^{2}-a^{2}(t)\left\{\frac{d r^{2}}{1-k r^{2}}+r^{2} d \theta^{2}+r^{2} \sin ^{2} \theta d \phi^{2}\right\},
$$

Eq.(đ) is nothing but the familiar Friedmann equation

$$
H_{G}=0 \rightarrow\left(\frac{\dot{a}}{a}\right)^{2}+\frac{k}{a^{2}}=\frac{8 \pi G}{3} \rho .
$$


As shown by deWitt in Ref.[1], the most general ultralocal supermetric is given by

$$
G_{i j k l} \equiv \frac{1}{2 \sqrt{h}}\left(h_{i k} h_{j l}+h_{i l} h_{j k}-\lambda h_{i j} h_{k l}\right) .
$$

The quantity $\lambda$ parametrizes the distance between metrics in superspace and, in principle, may take any real value except $\lambda=\frac{2}{3}$ for which the supermetric is singular.

When using (元) in Equation (4), and inserting a homogeneous and isotropic metric as in (5), one easily finds the following modified Friedmann equation

$$
\frac{1}{3 \lambda-2}\left(\frac{\dot{a}}{a}\right)^{2}+\frac{k}{a^{2}}=\frac{8 \pi G}{3} \rho
$$

This equation shows how the geometry of superspace percolates into the physics of the metric (5). As far as the geometry of superspace is concerned, there is no reason to have one or another value of $\lambda$; but as (8) demonstrates, the parameter $\lambda$ changes the expansion rate of the Universe and, thus, has observable cosmological implications.

In what follows, we will see how the $\lambda$-dependence in Eq.(8) can be understood as an effective modification to the number of internal degrees of freedom contributing to the density $\rho$ of the relativistic gas.

When we do not take superspace into account, the Friedmann equation for a radiation dominated Universe obtained from Eq.(6) is [4]

$$
H^{2}=\frac{8 \pi}{3 m_{p}^{2}} \rho=\frac{8 \pi}{3 m_{p}^{2}} \frac{\pi^{2}}{30} g_{*} T^{4} \rightarrow H \approx \frac{1.67}{m_{p}} g_{*}^{1 / 2} T^{2}
$$

where $g_{*}$ is defined by

$$
g_{*} \equiv \sum_{i=\text { fermions }} \frac{7}{8} g_{i}\left(\frac{T_{i}}{T}\right)^{4}+\sum_{i=\text { bosons }} g_{i}\left(\frac{T_{i}}{T}\right)^{4}
$$

and $g_{i}$ counts the number of internal degrees of freedom of the $i$-th species, $T$ is the photonic temperature and $T_{i}$ the temperature of the corresponding $i$-th species. When superspace effects are taken into consideration, we have seen that Eq. (9) has to be modified according to Eq.(8), and therefore Eq.(9) changes into

$$
H \approx \frac{1.67}{m_{p}} \sqrt{3 \lambda-2} g_{*}^{1 / 2} T^{2} \equiv \frac{1.67}{m_{p}} g_{*}^{1 / 2}(\lambda) T^{2}
$$


where in the last term we have redefined the effective $g_{*}$ as

$$
g_{*}(\lambda)=(3 \lambda-2)\left[\sum_{i=\text { fermiones }} \frac{7}{8} g_{i}\left(\frac{T_{i}}{T}\right)^{4}+\sum_{i=\text { bosones }} g_{i}\left(\frac{T_{i}}{T}\right)^{4}\right]
$$

so that, a general supermetric can be interpreted as inducing a redefinition of the parameter $g_{*}$ into an effective value which depends on $\lambda$.

If we take the number of neutrino species, $N_{\nu}$, to be 3 , as indicated by the CERN results [5], for temperatures of order of $M e V, g_{*}(\lambda)$ is

$$
g_{*}(\lambda)=\left.(3 \lambda-2)\left[2+\frac{7}{8} 2 N_{\nu}+\frac{7}{8} 2 \cdot 2\right]\right|_{N_{\nu}=3}=(3 \lambda-2) \times 10.75
$$

and since ${ }^{4} \mathrm{He}$ production is sensitive to the value of $g_{*}$, a change in $\lambda$ will change the amount of ${ }^{4} \mathrm{He}$ produced in the Big Bang nucleosynthesis.

We note the following general behavior: if $\lambda$ is equal to one, the standard results are recovered; if $\lambda$ is larger than one, the Big Bang nucleosynthesis produces more primordial ${ }^{4} \mathrm{He}$, and if $\lambda$ is smaller than one the amount of ${ }^{4} \mathrm{He}$ produced will be less than in the standard case.

Before we use the observational data on primordial abundances to place bounds on $\lambda$ via Eq.(11), we briefly discuss the available data on ${ }^{4} \mathrm{He}$ [6], $\mathrm{D}$, ${ }^{3} \mathrm{He}$ and ${ }^{7} \mathrm{Li}$ [7]].

It is possible to fit the observed ${ }^{4} \mathrm{He}$ abundance with respect to the abundances of $O$ and $N$ in $H_{I I}$-regions. The primordial ${ }^{4} \mathrm{He}$ corresponds to zero metallicity. A range consistent with observations is [6]

$$
Y_{P}=0.232 \pm 0.003 \pm 0.005
$$

where the first error is $1 \sigma$ statistical, and the second error is systematic (estimated at 2\%).

Through solar reactions, the initial amount (pre-solar) of $D$ is burned to form ${ }^{3} \mathrm{He}$, while the initial amount of ${ }^{3} \mathrm{He}$ is kept approximately constant in time. Therefore, the present ${ }^{3} \mathrm{He}$ abundance will be an indicator of the initial pre-solar, combined, $\mathrm{D}+{ }^{3} \mathrm{He}$ abundance. The former is measured in gas-rich meteorites [8], in the solar wind [9] and in the lunar soil [10], giving a value

$$
3.3 \times 10^{-5} \leq\left.\frac{N\left({ }^{3} H e\right)}{N(H)}\right|_{\text {grm }}\left(\left.\approx \frac{N\left({ }^{3} H e+D\right)}{N(H)}\right|_{\text {pre-solar }}\right) \leq 4.9 \times 10^{-5} .
$$


Obtaining primordial abundances from these pre-solar abundances is modeldependent, although it is possible to infer an upper bound on the primordial abundances of ${ }^{3} \mathrm{He}+\mathrm{D}$. This bound is 111, 12

$$
\left.\frac{N\left({ }^{3} H e+D\right)}{N(H)}\right|_{P} \leq 10^{-4}
$$

Furthermore, since carbonaceous chondrites (the oldest meteorites) are believed to provide a sample of pre-solar abundances, the fraction of ${ }^{3} \mathrm{He}$ measured in them gives us an estimate of pre-solar abundances of ${ }^{3} \mathrm{He}$ (without the solar contamination due to deuterium burning). These values are [13, 114]

$$
1.3 \times 10^{-5} \leq\left.\frac{N\left({ }^{3} H e\right)}{N(H)}\right|_{c c}\left(\left.\approx \frac{N\left({ }^{3} H e\right)}{N(H)}\right|_{\text {pre-solar }}\right) \leq 1.8 \times 10^{-5} .
$$

The difference between the pre-solar abundance of $D+{ }^{3} \mathrm{He}$ and the presolar abundance of ${ }^{3} \mathrm{He}$ will be the pre-solar abundance of deuterium, that is,

$$
1.8 \times 10^{-5} \leq\left.\frac{N(D)}{N(H)}\right|_{\text {pre-solar }} \leq 3.3 \times 10^{-5},
$$

and since deuterium is destroyed but is not produced (in significant quantities) in stellar processes, the minimum amount of deuterium in the pre-solar system gives us a lower bound on primordial deuterium. Thus

$$
\left.\frac{N(D)}{N(H)}\right|_{P} \geq\left.\frac{N(D)}{N(H)}\right|_{\text {pre-solar }} \geq 1.8 \times 10^{-5}
$$

Finally, for ${ }^{7} \mathrm{Li}$, very old Population II stars (halo stars) can provide an estimate of primordial ${ }^{7} \mathrm{Li}$ abundance. This hypothesis is supported by two observational facts: the plot of observational abundances of ${ }^{7} \mathrm{Li}$ versus iron metallicity has a plateau for low metallicity, implying that metal-poor stars do not produce significant amounts of ${ }^{7} \mathrm{Li}$; on the other hand, the plot of observational abundances of ${ }^{7} \mathrm{Li}$ versus effective temperature has another plateau for high temperatures, which implies that stars with high temperatures do not destroy their initial ${ }^{7} \mathrm{Li}$. 
For the hotter and more metal-poor Population-II stars in the halo, one has [7]

$$
\left.\left.\frac{N\left({ }^{7} \mathrm{Li}\right)}{N(H)}\right|_{P} \approx \frac{N\left({ }^{7} \mathrm{Li}\right)}{N(H)}\right|_{I I-\text { Pop }}=1.2 \pm 0.2 \times 10^{-10},
$$

so that an upper bound for primordial ${ }^{7} \mathrm{Li}$ is

$$
\left.\frac{N\left({ }^{7} L i\right)}{N(H)}\right|_{P} \leq 1.4 \times 10^{-10} .
$$

We summarize the above discussion in Table 1, where we also list the allowed values for $\eta_{10}$ obtained by comparing the observational data with theoretical standard nucleosynthesis; the neutron life-time $\tau_{1 / 2}$ has been taken between 10.19 and 10.35 minutes 1 and $N_{\nu}=3$. Notice that

$$
2.8 \leq \eta_{10} \leq 4.0
$$

is compatible [ with all the observational data.

We are now ready to derive bounds on $\lambda$ from the observed abundances of the light elements. Since the theoretical primordial mass fraction of ${ }^{4} \mathrm{He}$ is well fitted over the range $2.5 \leq \eta_{10} \leq 10$ by (Ref. [15]) the equation

$$
Y_{P}=0.228+0.010 \ln \left(\eta_{10}\right)+0.012\left(N_{\nu}-3\right)+0.017\left(\tau_{1 / 2}-10.27 \min \right)
$$

for temperatures of order $\mathrm{MeV}$, we obtain the following equation by using Eq.(13) in (24) to write $N_{\nu}$ as a function of $\lambda$ and then solving for $\lambda$,

$$
\lambda=1+4.52\left[\left(Y_{P}-0.228\right)-0.010 \ln \eta_{10}-0.017\left(\tau_{1 / 2}-10.27\right)\right] .
$$

Here $\tau_{1 / 2}$ must be given in minutes.

In Fig.1, we have plotted $\lambda$ versus $\eta_{10}$ (or, equivalently, $\Omega_{B} h_{100}^{2}$ ), with $Y_{P}$ and $\tau_{1 / 2}$ as parametric variables. As $\eta_{10}$ is increased, the parameter $\lambda$

\footnotetext{
${ }^{1}$ An accurate experiment to determine $\tau_{1 / 2}$ is described, e.g., in Ref. 116].

${ }^{2}$ This can also be expressed in terms of the combination $\Omega_{B} h_{100}^{2}$, where $\Omega_{B}$ is the baryonic fraction of the critical density and $h_{100}$ is the normalized Hubble parameter, as

$$
0.010 \leq \Omega_{B} h_{100}^{2} \leq 0.015 .
$$


decreases slowly since $\lambda$ evolves as a logarithm with $\eta_{10}$. The change in $\lambda$ with neutron lifetime is also small for the allowed band in $\tau_{1 / 2}$.

The most pronounced effect of $\lambda$ shows up in the ${ }^{4} H e$ abundance. When $\lambda$ is increased, the amount of ${ }^{4} \mathrm{He}$ also increases. Therefore $\lambda$ can substantially disturb the Big-Bang nucleosynthesis]. This can be used in two different ways: the observations of primordial ${ }^{4} \mathrm{He}$ give bounds on the values of $\lambda$ or, if the observational data on primordial ${ }^{4} \mathrm{He}$ are too low, a value of $\lambda$ smaller than 1 helps to bring back consistence into Big-Bang nucleosynthesis.

Concerning the first point, we could let $Y_{P}, \tau_{1 / 2}$ and $\eta_{10}$ vary inside their bounds, to give a range for $g_{*}$ and from there deduce upper and lower bounds on $\lambda$. For the extreme values quoted in Table 1 ,

$$
\begin{aligned}
0.221 & \leq Y_{P} \leq 0.243 \\
10.19 \mathrm{~min} & \leq \tau_{1 / 2} \leq 10.35 \mathrm{~min} \\
2.8 & \leq \eta_{10} \leq 4.0
\end{aligned}
$$

Using equation (24), one finds that

$$
7.5 \leq g_{*} \leq 11.6 \quad\left(1.1 \leq N_{\nu} \leq 3.5\right)
$$

If we consider the particle contents in the standard model, these bounds over $g_{*}$ imply

$$
7.5 \leq(3 \lambda-2) \cdot 10.75 \leq 11.6,
$$

which translates into the following range for $\lambda$,

$$
0.90 \leq \lambda \leq 1.03
$$

The bounds on $\lambda$ depend on the "choice" of observational bounds on $Y_{P}, \tau_{1 / 2}$ and $\eta_{10}$. To see this, we select less restrictive values for $Y_{P}$ [7]: $0.215 \leq Y_{P} \leq 0.245$. The bounds over $\lambda$ now translate into f

$$
0.87 \leq \lambda \leq 1.04
$$

\footnotetext{
${ }^{3}$ We are going to analyze the influence of $\lambda$ only on ${ }^{4} \mathrm{He}$ production. It can be seen that the effect of $\lambda$ on the remaining light elements is negligible compared with observational errors.

${ }^{4}$ These bounds agree with the bounds in Ref. 20].
} 
and therefore the bounds do not change very much for different observational data, with the values of $\lambda$ always near 1 .

On the other hand, recent observational and theoretical results on ${ }^{4} \mathrm{He}$ abundances could be in conflict with the abundances for the remaining light elements, and thus put in danger the consistency of the standard Big-Bang nucleosynthesis [6]. A more accurate theoretical calculation [17, 18], increases the value of $Y_{P}$ with respect to the results of Ref. [7]. Furthermore, the present errors on $\tau_{1 / 2}$ have been slightly reduced, and $\tau_{1 / 2}=10.27 \pm 0.024$ [19]. These two effects combine and make slightly more difficult the agreement between observational and theoretical values, with the net effect of increasing the discrepancy.

In Fig. $2, Y_{P}$ is plotted versus $\lambda$, with the previous considerations already taken into account. The horizontal dashed lines correspond to the observations on $Y_{P}$ [6]. We have plotted the average $Y_{P}$ and the upper bounds at $1 \sigma, 2 \sigma$ and $2 \sigma+\sigma_{\text {sys }}$.

For the average $Y_{P}$, standard Big-Bang nucleosynthesis $(\lambda=1$, vertical solid line) is incompatible with the observations of the other light elements, which fall between the two extreme dotted lines $\left(\eta_{10}=4.0, \tau_{1 / 2}=10.32\right.$ and $\left.\eta_{10}=2.8, \tau_{1 / 2}=10.22\right)$. The incompatibility is not removed for observational values of $Y_{P}$ inside of the bounds for $1 \sigma$-errors or $2 \sigma$-errors. To recover consistency, systematic errors in observational data must be considered. However, consistency between observation and theory is recovered if we consider values of $\lambda$ away from 1 . For the average value of $Y_{P}, Y_{P}=0.232$, we obtain $\lambda \approx 0.95$. With $\lambda \lesssim 0.98$ we are inside of the region of observational errors on $Y_{P}$ less than or equal to $1 \sigma$, and with $\lambda \lesssim 0.99$ we are inside of the region of $2 \sigma$ errors. Thus

$$
\begin{gathered}
0.93 \leq \lambda \leq 0.98 \quad(1 \sigma) \\
0.91 \leq \lambda \leq 0.99 \quad(2 \sigma) \\
0.89 \leq \lambda \leq 1.01 \quad\left(2 \sigma+\sigma_{\text {sys }}\right)
\end{gathered}
$$

for errors in observational $Y_{P}$ of $1 \sigma, 2 \sigma$ and $2 \sigma+\sigma_{\text {sys }}$ respectively?.

Therefore with $\lambda<1$ the Big-Bang nucleosynthesis is absolutely consistent. Future, more accurate observations, should clarify the confluence or

\footnotetext{
${ }^{5}$ These last values were obtained using the ${ }^{4} \mathrm{He}$ production from Ref. 17, 18, whereas for the previous bounds given in Eq.(31), Ref.[7] was used.
} 
inconsistence in the observations of the abundances of all light elements. If the present situation is confirmed, $\lambda$ could be used to explain these discrepancies.

Finally we offer some conclusions. First we note that there exists a connection between the physics of superspace and nucleosynthesis due to the fact that $\lambda$ contributes to the Friedmann equation in a way which can be interpreted as a modification to the effective number of degrees of freedom of the relativistic gas which dominates the energy density of the Universe during the Nucleosynthesis era; this has as a consequence that as $\lambda$ increases, so does $Y_{P}$.

Using the observational data on primordial ${ }^{4} \mathrm{He}, \mathrm{D},{ }^{3} \mathrm{He}$ and ${ }^{7} \mathrm{Li}$, we have also obtained bounds on $\lambda$. These bounds depend on the values considered for $Y_{P}, \tau_{1 / 2}$ and $\eta_{10}$, but they tend to cluster near $\lambda=1$. A reasonable range of values for $\lambda$ is

$$
0.87 \leq \lambda \leq 1.04
$$

From the point of view of superspace, therefore, nucleosynthesis implies that the deviation from standard $\operatorname{FRW}(\lambda=1)$ is small. But if these deviations exist, although small, they could be useful: as present data indicates, measurements of primordial ${ }^{4} \mathrm{He}$ could be inconsistent at the $2 \sigma$ level with the remaining observed abundances of $\mathrm{D},{ }^{3} \mathrm{He}$ and ${ }^{7} \mathrm{Li}$, having a slightly low value (if systematic errors are considered, consistence can be recovered). If the errors in the observations decrease in the future, and the value of the ${ }^{4} \mathrm{He}$ abundance was too small, and inconsistent with the abundances of the other light elements, a value of $\lambda$ less than 1 could be used to understand this disagreement; this brings with it a slight deviation from the cosmological standard model at the time of nucleosynthesis.

On the other hand a value of $\lambda$ different from one, has other observational cosmological effects, such as modifications on the spectrum of the density perturbations or on the microwave background anisotropies, in addition to alterations in the evolution of the angular sizes of luminous sources with red-shift. These effects will be considered in a separate paper.

\section{ACKNOWLEDGMENTS}

We thank Domenico Giulini for stimulating discussions. This work has been supported in part by INTA and DGICyT Funds. 


\section{References}

[1] B. S. deWitt, Phys. Rev. 160, 1113 (1967).

[2] J. F. Barbero and J. Pérez-Mercader, Phys. Rev. D 48, 3663 (1993).

[3] R. Arnowitt, S. Deser and C. W. Misner, The dynamics of general relativity, in Gravitation (ed. L.Witten, Wiley, New York, 1962), p. 227-265.

[4] E. W. Kolb and M. S. Turner, in The Early Universe (Addison-Wesley, 1990), p. 64.

[5] F. Dydak, in Proc. 25th Int. Conf. on High Energy Physics (ed. Rhua, World Scientific, Singapore, 1991).

[6] K. A. Olive and G. Steigman, astro-ph/9405022, preprint (1994).

[7] T. P. Walker, G. Steigman, D. N. Schramm, K. A. Olive and H. Kang, Ap.J. 376, 51 (1991).

[8] P. M. Jeffrey and E. Anders, Geochim. Cosmochim. Acta 34, 1175 (1970).

[9] J. Geiss, P. Eberhardt, F. Buhler, J. Meister and P. Singer, J. Geophys. Res. 75, 5972 (1970).

[10] D. C. Black, Geochim. Cosmochim. Acta 36, 347 (1972).

[11] J. Yang, M. S. Turner, G. Steigman, D. N. Schramm and K. A. Olive, Ap.J. 281, 493 (1984).

[12] K. A. Olive, D. N. Schramm, G. Steigman and T. P. Walker, Phys. Lett. B, 236, 454 (1990).

[13] P. Eberhardt, in Proc. 9th Lunar Planet. Sci. Conf., 1027 (1978).

[14] U. Frick and R. K. Moniot, in Proc. 8th Lunar Planet. Sci. Conf., 229 (1977).

[15] K. A. Olive, D. N. Schramm, G. Steigman and T. P. Walker, Phys. Lett. B 236, 454 (1990). 
[16] W. Mampe, P. Ageron, C. Bates, J. M. Pendebury and A. Steyerl, Phys. Rev. Lett. 63A, 593 (1989).

[17] P. Kernan, OSU Physics Ph. D. Thesis (1993).

[18] P. Kernan and L. Krauss, Preprint astro-ph/9402010, to appear in Phys. Rev. Lett..

[19] Review of Particle Properties, Phys. Rev. D 45, Part 2 (June 1992).

[20] D. Giulini and C. Kiefer, Preprint gr-qc/9405040. 
Figure 1: $\lambda$ versus $\eta_{10}\left(\right.$ or $\left.\Omega_{B} h_{100}^{2}\right)$. We have considered the values $Y_{P}=$ $0.221,0232,0.243$. The solid lines correspond to $\tau_{1 / 2}=10.27$. The dashed lines are associated to the uncertainties in $\tau_{1 / 2}$ : for each $Y_{P}$, the top dashed line is $\tau_{1 / 2}=10.19$ and the bottom dashed line is $\tau_{1 / 2}=10.35$ [7]. For $Y_{P}$ fixed, $\lambda$ increases as $\Omega_{B}$ decreases, and for $\Omega_{B}$ fixed, $\lambda$ rises the production of ${ }^{4} \mathrm{He}$.

Figure 2: Production of ${ }^{4} \mathrm{He}$ versus $\lambda$. The solid lines correspond to $\eta_{10}=2.8$ and $\eta_{10}=4.0$ (interval allowed by observations for the abundances of ${ }^{3} \mathrm{He}, \mathrm{D}$ and ${ }^{7} \mathrm{Li}$ ) with $\tau_{1 / 2}=10.27 \mathrm{~min}$. The dotted lines represent the $2 \sigma$ limits on $\tau_{1 / 2}[19]: \tau_{1 / 2}=10.22$ and $\tau_{1 / 2}=10.32$ (for each $\eta_{10}$ ). The horizontal dashed lines correspond to the observational values [6]: average $Y_{P}$ and upper bounds $1 \sigma, 2 \sigma$ and $2 \sigma+\sigma_{\text {sys }}$ ( $\sigma$ is statistical error and $\sigma_{\text {sys }}$ systematic error). It is clear that the standard Big-Bang nucleosynthesis $(\lambda=1$, vertical solid line) is not compatible inside the $1 \sigma$ and $2 \sigma$ errors. It is neccesary to consider systematic errors. The compatibility is immediately recovered if we consider a value of $\lambda$ away from 1 . 


\begin{tabular}{|c|c|c|c|c|}
\hline 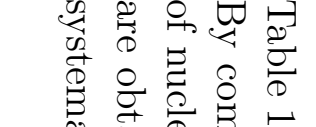 & & OBSERVATIONAL ABUNDANCES & BOUNDS & $\eta_{10}$ \\
\hline 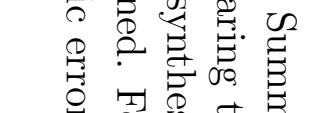 & ${ }^{4} \mathrm{He}$ & $Y_{P}=0.232 \pm 0.003 \pm 0.005$ & $Y_{P} \leq 0.243$ & $\eta_{10} \leq 5.1$ \\
\hline 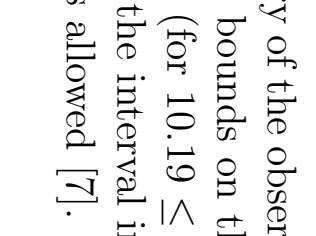 & $\begin{array}{c}D+{ }^{3} \mathrm{He} \\
{ }^{3} \mathrm{He}\end{array}$ & $\begin{array}{c}3.3 \times 10^{-5} \leq\left.\frac{N\left(^{3} H e+D\right)}{N(H)}\right|_{\text {pre-solar }} \leq 4.9 \times 10^{-5} \\
1.3 \times 10^{-5} \leq\left.\frac{N\left({ }^{3} H e\right)}{N(H)}\right|_{\text {pre-solar }} \leq 1.8 \times 10^{-5}\end{array}$ & $\left.\frac{N\left({ }^{3} H e+D\right)}{N(H)}\right|_{P} \leq 1 \times 10^{-4}$ & $\eta_{10} \geq 2.8$ \\
\hline 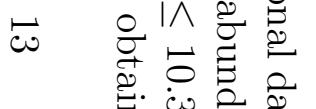 & $D$ & $1.8 \times 10^{-5} \leq\left.\frac{N(D)}{N(H)}\right|_{\text {pre-solar }} \leq 3.3 \times 10^{-5}$ & $\left.\frac{N(D)}{N(H)}\right|_{P} \geq 1.8 \times 10^{-5}$ & $\eta_{10} \leq 6.8$ \\
\hline 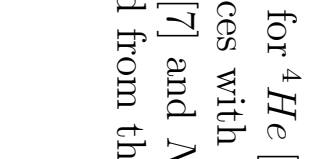 & ${ }^{7} \mathrm{Li}$ & $\left.\frac{\left.N^{(7} L i\right)}{N(H)}\right|_{I I-P o p}=1.2 \pm 0.2 \times 10^{-10}$ & $\left.\frac{\left.N^{(7} L i\right)}{N(H)}\right|_{P} \leq 1.4 \times 10^{-10}$ & $1.6 \leq \eta_{10} \leq 4.0$ \\
\hline 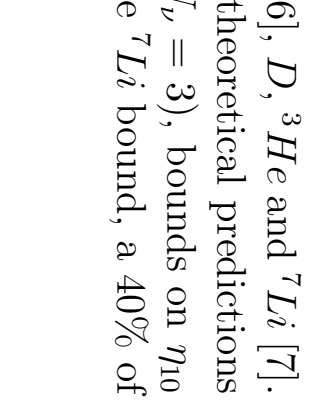 & & & & \\
\hline
\end{tabular}




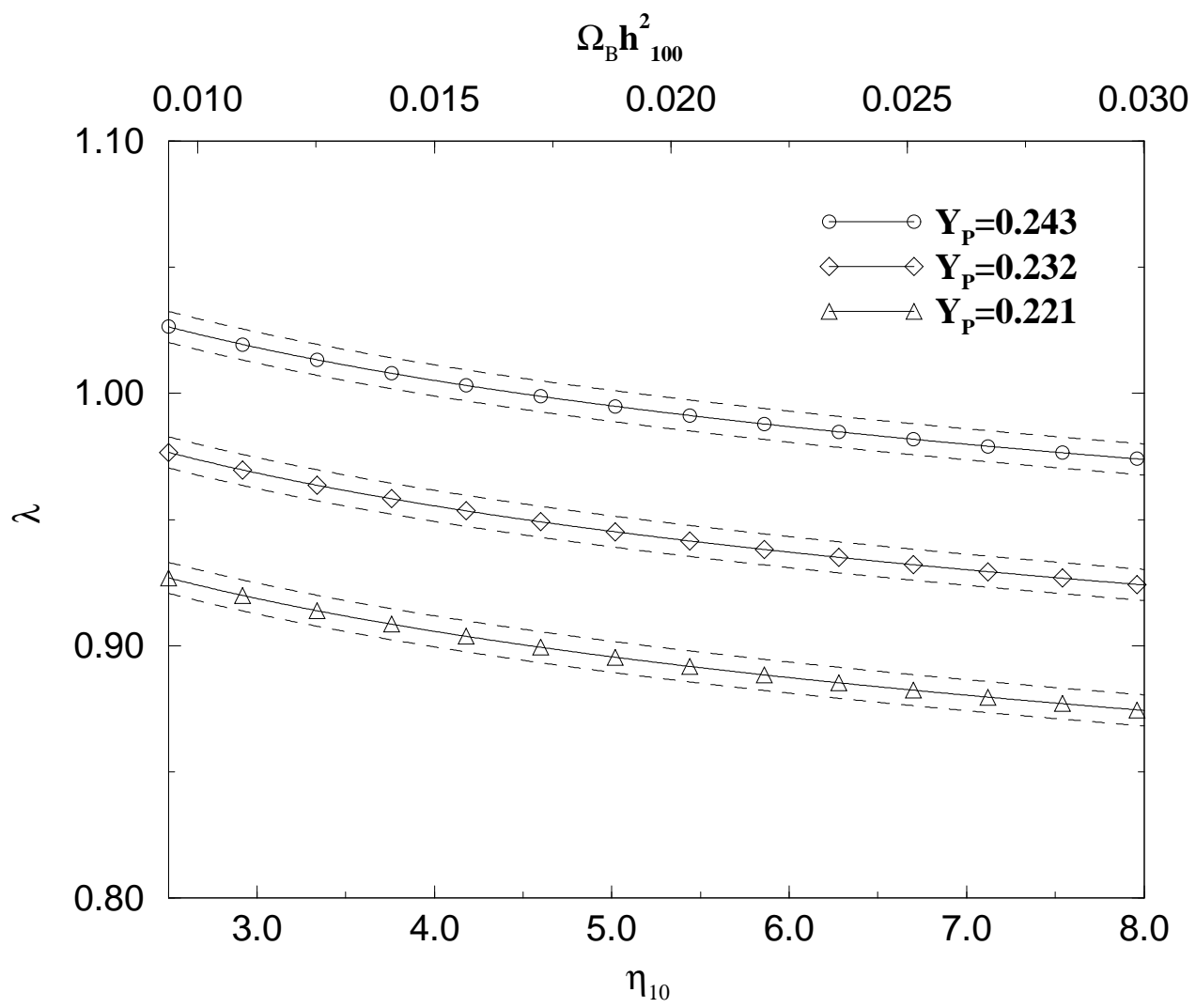

Fig. 1 


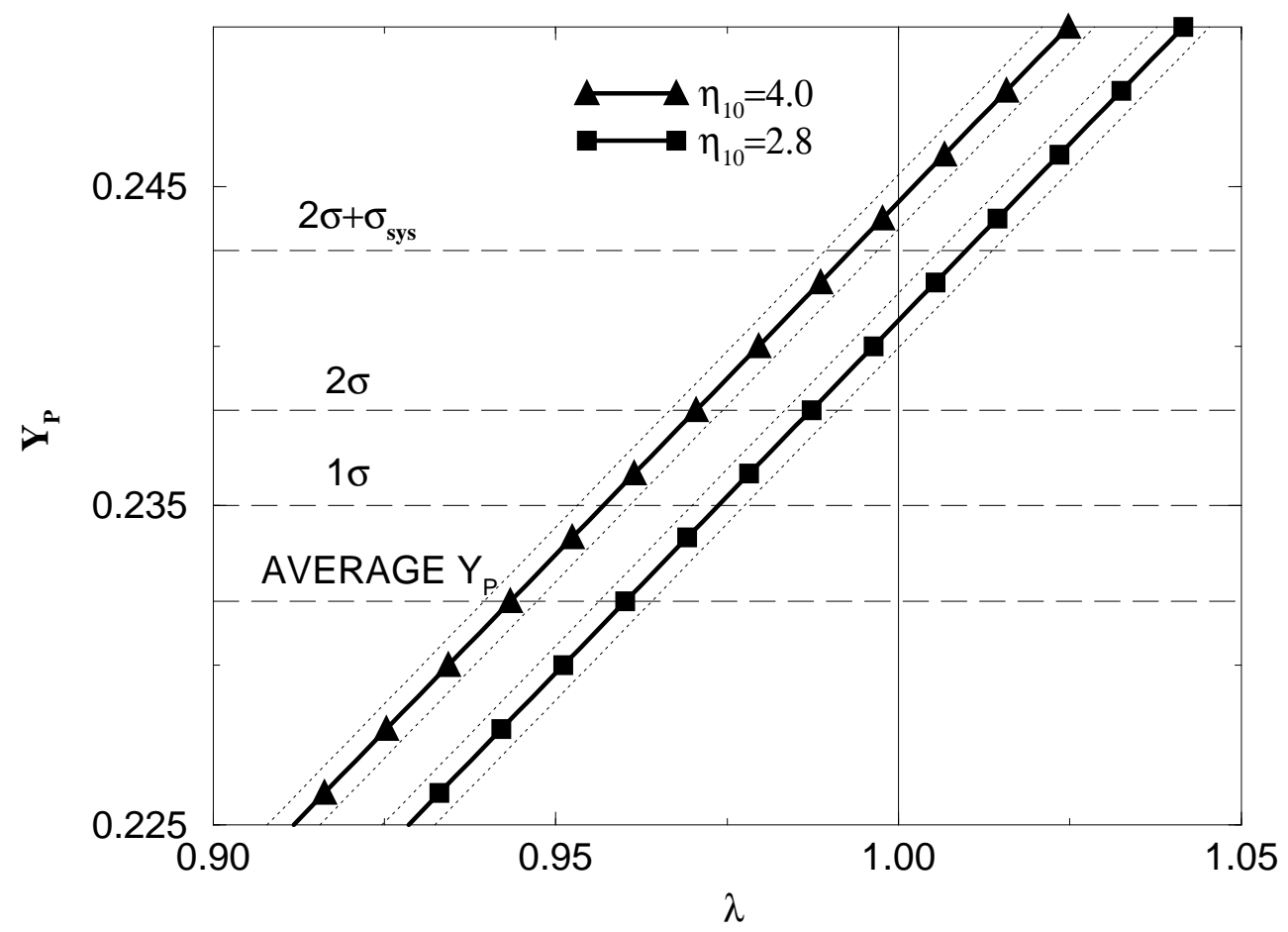

Fig. 2 
This figure "fig1-1.png" is available in "png" format from: http://arxiv.org/ps/astro-ph/9412094v2 
This figure "fig1-2.png" is available in "png" format from: http://arxiv.org/ps/astro-ph/9412094v2 